\title{
Evolving surgical techniques for pulmonary endarterectomy according to the changing features of chronic thromboembolic pulmonary hypertension patients during 17-year single-center experience
}

\author{
Marco Morsolini, MD ${ }^{\mathrm{a}, \mathrm{b}}$ Salvatore Nicolardi, MD, ${ }^{\mathrm{a}, \mathrm{b}}$ Elisa Milanesi, MD, ${ }^{\mathrm{c}}$ Eleonora Sarchi, MD, \\ Gabriella Mattiucci, MD, ${ }^{\mathrm{a}}$ Catherine Klersy, MD, MSc, ${ }^{\mathrm{e}}$ and Andrea Maria D'Armini, $\mathrm{MD}^{\mathrm{a}}$
}

\begin{abstract}
Objective: An increasing number of elderly patients are referred for pulmonary endarterectomy. The distinction between operable and inoperable lesions has been challenged over time. Hence, we developed alternative cardiopulmonary bypass management and cerebral protection strategies to obtain satisfactory surgical results according to the changing patient features.
\end{abstract}

\begin{abstract}
Methods: From April 1994 to March 2011, 347 pulmonary endarterectomies were performed at our center. We began with the technique championed by the San Diego Group, adopting a single period of deep hypothermic circulatory arrest for each side (group A). Since 2003, we began to perform short periods of intermittent deep hypothermic circulatory arrest followed by periods of reperfusion (group B). We then adopted moderate, instead of deep, hypothermia (group C). Finally, we modified our technique further performing shorter (5-7-minute) periods of circulatory arrest (group D).

Results: The hemodynamic results after surgery were excellent in all 4 groups. The patients' age increased significantly. A trend toward an increase in the number of Jamieson type 3 lesions was observed. Associated with our protocol changes, we observed better postoperative respiratory function, a reduction in the length of mechanical ventilation and postoperative infections, and a remarkable improvement in uneventful postoperative courses. Despite the increased total circulatory arrest time, a trend toward a reduction in the incidence of transient neurologic events was observed, and operative mortality was not affected.
\end{abstract}

Conclusions: In our experience, our alternative strategy resulted in a better combination of surgical accuracy and cerebral protection and improved outcomes. (J Thorac Cardiovasc Surg 2012;144:100-7)

Chronic thromboembolic pulmonary hypertension (CTEPH) is a progressive disease due to the incomplete resolution of pulmonary emboli, leading to right heart failure, with a poor survival if left untreated. Despite the improved prognosis with the introduction of specific medical treatment, ${ }^{1,2}$ pulmonary endarterectomy (PEA) is the only curative therapy ${ }^{1-9}$ able to significantly improve the normally poor prognosis. ${ }^{10}$ Even if appropriate medical therapy is promptly started, CTEPH is estimated to occur in approximately $0.5 \%$ to $3.8 \%$ of patients who survive an acute episode of pulmonary embolism. ${ }^{11,12}$ The true incidence is suspected to be

From the Division of Cardiac Surgery, ${ }^{\mathrm{a}}$ Department of Surgical Sciences, ${ }^{\mathrm{b}}$ Department of Anesthesiology and Intensive Care Unit III, ${ }^{\mathrm{C}}$ Department of Infectious Diseases, ${ }^{\mathrm{d}}$ and Service of Biometry and Clinical Epidemiology, ${ }^{\mathrm{e}}$ University of Pavia School of Medicine, Foundation "I.R.C.C.S. San Matteo" Hospital, Pavia, Italy. Disclosures: Authors have nothing to disclose with regard to commercial support.

Read at the 37th Annual Meeting of The Western Thoracic Surgical Association, Colorado Springs, Colorado, June 22-25, 2011.

Received for publication June 21, 2011; revisions received Oct 11, 2011; accepted for publication Nov 8, 2011; available ahead of print April 12, 2012.

Address for reprints: Marco Morsolini, MD, Division of Cardiac Surgery, University of Pavia School of Medicine, Foundation "I.R.C.C.S. San Matteo" Hospital, Piazzale Camillo Golgi, Pavia 2-27100, Italy (E-mail: m.morsolini@smatteo.pv.it).

$0022-5223 / \$ 36.00$

Copyright (c) 2012 by The American Association for Thoracic Surgery doi:10.1016/j.jtcvs.2011.11.041 much greater, ${ }^{13}$ because small pulmonary emboli would not be noticed unless many of them were showered into the pulmonary circulation at once or within a certain period. Hence, the epidemiology of CTEPH remains uncertain. Although CTEPH is currently gaining the interest of a large number of physicians and is increasingly being diagnosed, underdiagnosis and late diagnosis remain the main issues. ${ }^{3}$ Surgery is indicated as soon as it has been diagnosed, before the typical arteriopathy develops in both obstructed and nonobstructed branches. ${ }^{214-16}$ PEA avoids the onset of the described vascular remodeling, which is responsible for the progressive worsening of CTEPH and the development of a reactive component of pulmonary hypertension, not directly curable by surgery.

Because no well-defined criteria are available to discriminate proximal from distal obstructive lesions, a patient should not be considered inoperable unless the case has been reviewed by an experienced surgeon. ${ }^{2,3,16}$ According to recent guidelines, a center can be considered to have sufficient expertise if the number of PEAs performed is at least 20 annually, with a mortality rate less than $10 \% .{ }^{16} \mathrm{Be}-$ cause advanced techniques that enable dissection at subsegmental level have been developed, no embolic obstruction is inaccessible, according to the Jamieson operative 


\section{Abbreviations and Acronyms \\ $\mathrm{CPB}=$ cardiopulmonary bypass \\ cNIRS $=$ cerebral near-infrared spectroscopy \\ $\mathrm{CTEPH}=$ chronic thromboembolic pulmonary hypertension \\ HCA $=$ hypothermic circulatory arrest \\ $\mathrm{J} 3=$ Jamieson type 3 \\ LMV = length of mechanical ventilation \\ $\mathrm{PaO}_{2} / \mathrm{FiO}_{2} 6 \mathrm{~h}=$ partial pressure of oxygen in arterial blood/fraction of inspired oxygen 6 hours after admission to intensive care unit \\ PEA $\quad=$ pulmonary endarterectomy \\ PVR = pulmonary vascular resistance}

classification of CTEPH lesions. ${ }^{17}$ Thus, no grades of right ventricular failure or levels of pulmonary vascular resistance (PVR) contraindicate PEA. ${ }^{18,19}$ However, the prognosis worsens with greater preoperative pulmonary pressure and greater postoperative PVR. ${ }^{3,19,20}$

The surgical technique was largely developed by Jamieson. ${ }^{7}$ The complete removal of all thromboembolic materials is the key factor for a satisfactory outcome. To achieve a completely bloodless surgical field, deep hypothermic circulatory arrest (HCA) with aortic crossclamping has been promoted as the strategy of choice and is still used in the vast majority of centers performing PEA worldwide. ${ }^{3}$ PEA is still thought to be a very invasive surgery because of the deep HCA required. Alternative cerebral protection strategies have recently been proposed, especially concerning PEA in the elderly, causing intense debate on the need for deep HCA. ${ }^{21,22}$ At our center, we started in 1994 to adopt the San Diego technique based on deep HCA, except for leaving the aorta unclamped. As we gained experience, we changed our cardiopulmonary bypass (CPB) management further, adapting it to our evolving learning curve, to minimize the burden of HCA. In the present study, we analyzed the postoperative outcomes according to the different strategies used.

\section{METHODS}

\section{Patient Selection}

From April 1994 to March 2011, 347 consecutive patients diagnosed with CTEPH underwent PEA at our center. From 1994 onward, a prospective dedicated database, approved by the institutional review board, recorded the patients' information. All patients provided informed consent. Except for urgent or emergency operations, all CTEPH patients admitted to our center underwent a complete diagnostic workup, according to our published standardized protocol. ${ }^{23,24}$ The operability assessment was performed by the same surgeon. A permanent inferior vena cava filter was usually placed the day before surgery, unless contraindicated.

At our center, the only contraindication to PEA is underlying severe parenchymal lung disease. Some investigators also consider the severity of hemodynamic impairment, underlying major comorbidities, and patient age. $^{2-6,18,19}$ In our experience, we have never excluded any patient from surgery on the basis of only age and preoperative conditions.

Patient referrals increased substantially over the years, and we became more confident with the procedure. During 2004, 54 patients were referred to our center for a surgical evaluation. A CTEPH diagnosis was confirmed in 34 patients $(63 \%)$, and the disease was deemed operable in 25 , for an operability rate of $74 \%$. During 2010 , we evaluated 108 patients, and the diagnostic accuracy for CTEPH of the referring centers had increased to $76 \%$ (82/108 patients). Owing to our learning curve, CTEPH was deemed operable in 73 patients. Thus, operability rate increased from $74 \%$ to $89 \%$ (73/82). This resulted in a significant increase in PEAs performed each year (Figure 1).

\section{Surgical Technique}

At our center, the surgical approach is according to the published surgical technique, ${ }^{7}$ except for some technical details. The right atrium is explored only if any atrial septal defect or thrombosis of the right heart chambers is demonstrated during the diagnostic workup or by intraoperative transesophageal echocardiography. The aorta is left unclamped to avoid excessive manipulation of potential atherosclerotic plaques. Myocardial protection is achieved without cardioplegia administration, exclusively by hypothermia, with the left ventricle vented, even in the presence of any degree of aortic regurgitation. The left ventricular vent is placed after hypothermic ventricular fibrillation has occurred. An electric 2-lead cardiac fibrillator is placed when fibrillation does not occur despite hypothermic perfusion. In ideal conditions, PEA can generally be extended even toward the fourth and fifth order of arterial bifurcation. The operation must always be performed bilaterally, because sometimes the imaging tools can fail to detect partially obstructed or recanalized arteries or intraluminal webs, which are almost regularly perfused by contrast agent. Once PEA is completed, the left ventricular vent is clamped and bronchoscopy is performed to assess the absence of airway bleeding. At the end of the operation, the pericardium is closed to reduce the development of adhesions and promote better postoperative right ventricular function.

The following associated cardiac procedures were performed: 22 coronary artery bypass grafts, 14 atrial septal defect repairs, and 2 aortic valve replacements.

\section{CPB Management}

Our cerebral protection strategy has changed over time (Table 1). At the beginning of our experience, we used to perform a single period of deep HCA for each side (group A, 83 patients). In 2003, we performed a PEA reoperation in a 80-year-old male patient. Five sessions of deep HCA of 20 minutes and one of 15 minutes were necessary. The hemodynamic result after PEA was excellent; however, the patient died of massive intestinal ischemia, probably as a complication of the long HCA period on his intestinal microcirculation with a reduced flow reserve. Since then, we changed our CPB management to reduce the burden of HCA, especially in the elderly. Hence, we started to perform shorter (10-15-minute) periods of intermittent deep HCA followed by short ( $\geq 5$-minute) periods of reperfusion. We used cerebral near-infrared spectroscopy (cNIRS) to determine the length of each reperfusion period (group B, 70 patients). Given evidence that we could safely prolong the total HCA time, we started to thoroughly explore the segmental branches found to be patent on preoperative examination. In some patients, we were able to find and remove unexpected lesions, such as recanalized thrombi and webs. To further reduce the surgical invasiveness, we combined intermittent periods of HCA with moderate, instead of deep, hypothermia (group C, 91 patients). The repeated interruptions during PEA for reperfusion did not cause any significant loss of time and did not represent a problem for the surgeon. Therefore, we modified our technique further, performing even shorter (5-7-minute) periods of moderate HCA (group D, 103 patients). 


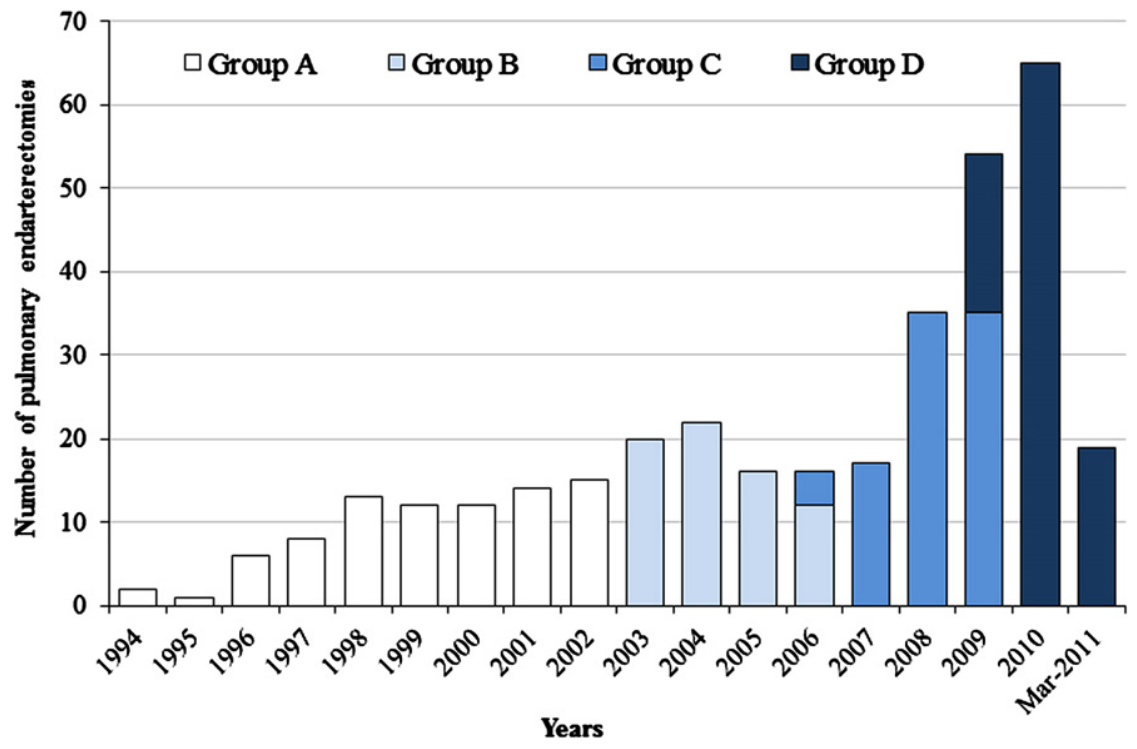

FIGURE 1. Pulmonary endarterectomies (PEAs) performed by year and group.

\section{Anesthesia Management}

All the patients were monitored according to our protocol, including radial and femoral arterial lines for blood pressure, a Swan-Ganz catheter, rectal and esophageal temperature probes, electrocardiography, pulse oximetry, expired end-tidal carbon dioxide, transesophageal echocardiography, and electroencephalography. cNIRS monitoring was adopted for group B onward. Once CPB has been established, the patient's systemic temperature is cooled and hemodilution performed until the hemoglobin reaches $7 \mathrm{~g} / \mathrm{dL}$. In addition, a cooling helmet is placed. When the target temperature is reached, thiopental is administered until the electroencephalogram becomes isoelectric. The bilateral cNIRS values are noted as the baseline and compared during the circulatory arrests. Particular attention must be paid to both the rate of the cNIRS decrease during HCA and the rate of the cNIRS increase during reperfusion. Norepinephrine and dobutamine (or epinephrine) are routinely administered to assist with weaning from $\mathrm{CPB}$ by improving the systemic perfusion pressure, including the coronary blood flow. Inhaled nitric oxide is generally administered to improve right ventricular function, especially if the PVR is not yet back to normal.

\section{Early Postoperative Management}

After PEA, the cardiac output is generally high, owing to the remarkable unloading of the hypertrophic right ventricle, and can lead to lung reperfusion edema. Hence, inotropes and inhaled nitric oxide are rapidly discontinued, vasopressors agents are continued for a few days, and vigorous diuresis is achieved to obtain a negative fluid balance. Protective ventilation (high positive end-expiratory pressure and low tidal volume) is preferred to prevent both lung reperfusion edema and ventilation-induced lung injury. Extubation is performed as soon as possible, switching to helmet continuous positive airway pressure ventilation for a few days. Vancomycin and ceftazidime are routinely administered, unless other antibiotics are needed according to the antibiogram.

\section{Statistical Analysis}

Our research was a retrospective case-cohort study with noncontemporaneous cohorts. The patient characteristics are reported as counts and percentages, if categorical, and as the mean \pm standard deviation or median and 25 th to 75 th percentiles, if continuous. The variables were compared among the 4 different operative protocols using the Fisher exact test and analysis of variance (either parametric or nonparametric, eg, the KruskalWallis test) if continuous. The paired Student $t$-test was used to compare the preoperative and postoperative values of the mean pulmonary arterial pressure and PVR.

The association between the operative protocol and outcome was assessed using generalized linear regression models (with either identity or logistic link). Furthermore, the independent role of CPB management on the operative outcome was controlled in a multivariate model for other potential predictors of outcome (identified a priori, from the published data, and including age, duration of severe disease, Jamieson type 3 (J3) CTEPH lesions, preoperative mean pulmonary arterial pressure, PVR, and PEA surgical timing). As outcome endpoints, we considered the following continuous variables: partial pressure of oxygen in arterial blood/fraction of inspired oxygen 6 hours after admission to intensive care unit $\left(\mathrm{PaO}_{2} /\right.$ $\mathrm{FiO}_{2} 6 \mathrm{~h}$ ) ratio, length of mechanical ventilation (LMV), and total intensive care unit stay. The following categorical variables were considered: reperfusion edema, need for tracheostomy, any infection, transient neurologic events, operative mortality, and hospital mortality. Also, the $\mathrm{PaO}_{2} / \mathrm{FiO}_{2}$ $6 \mathrm{~h}$ ratio, LMV, and total intensive care unit stay were considered when dichotomized according to the worst tertile (lower for $\mathrm{PaO}_{2} / \mathrm{FiO}_{2}$ 6h and

TABLE 1. Study population by group according to cardiopulmonary bypass management and cerebral protection strategy

\begin{tabular}{lll}
\hline Group & \multicolumn{1}{c}{ CPB management } & \multicolumn{1}{c}{ Cerebral protection strategy* } \\
\hline A & Single 20-min HCA for each PEA side & Deep HCA \\
B & Intermittent 10-15-min HCA interrupted by reperfusion periods & Deep HCA with cNIRS monitoring \\
C & Intermittent 10-15-min HCA interrupted by reperfusion periods & Moderate HCA with cNIRS monitoring \\
D & Intermittent 7-min HCA interrupted by reperfusion periods & Moderate HCA with cNIRS monitoring \\
\hline
\end{tabular}

$C P B$, Cardiopulmonary bypass; $H C A$, hypothermic circulatory arrest; PEA, pulmonary endarterectomy; $c N I R S$, cerebral near-infrared spectroscopy. *Pharmacologic protocol and anesthesia management were the same for all groups. 
TABLE 2. Patient preoperative and operative characteristics

\begin{tabular}{|c|c|c|c|c|c|c|c|}
\hline Characteristic & Overall & Group A & Group B & Group C & Group D & $\begin{array}{l}\text { Overall } \\
P \text { value } \\
\end{array}$ & $\begin{array}{c}\text { Significant post hoc } \\
\text { comparisons with Bonferron } \\
\text { correction: } P \text { value }<.008\end{array}$ \\
\hline \multicolumn{8}{|l|}{ Preoperative } \\
\hline Age $(y)$ & $57 \pm 16$ & $52 \pm 15$ & $56 \pm 17$ & $60 \pm 15$ & $60 \pm 16$ & $<.001$ & A vs $C$ and $D$ \\
\hline Age $\geq 70 y$ & $95(27.4)$ & $9(10.8)$ & $16(22.9)$ & $31(34.1)$ & $39(37.9)$ & $<.001$ & A vs $C$ and $D$ \\
\hline Male gender & $168(48.4)$ & $47(56.6)$ & $36(51.4)$ & $41(45.1)$ & $44(42.7)$ & .27 & - \\
\hline \multicolumn{8}{|l|}{$\operatorname{LOSD}(\mathrm{m})$} \\
\hline Median & 12 & 13 & 17 & 9 & 11 & .005 & $\mathrm{~B}$ vs $\mathrm{C}$ \\
\hline Interquartile range & $6-24$ & $8-24$ & $9-30$ & $5-19$ & $4-24$ & & \\
\hline J3 lesions & $93(26.8)$ & $18(21.7)$ & $19(27.1)$ & $24(26.4)$ & $32(31.1)$ & .58 & - \\
\hline mPAP $(\mathrm{mmHg})$ & $45 \pm 13$ & $50 \pm 13$ & $46 \pm 12$ & $41 \pm 12$ & $43 \pm 11$ & $<.001$ & A vs $C$ and $D$ \\
\hline PVR (dyne $\cdot \mathrm{s} \cdot \mathrm{cm}^{-5}$ ) & $999 \pm 482$ & $1214 \pm 485$ & $1060 \pm 549$ & $877 \pm 421$ & $897 \pm 419$ & $<.001$ & $A$ vs $C$ and $D$ \\
\hline Oxygen therapy & $168(48.4)$ & $39(47.0)$ & $38(54.3)$ & $44(48.4)$ & $47(45.6)$ & .75 & - \\
\hline Urgent/emergency & $76(21.9)$ & $28(33.7)$ & $20(28.6)$ & $13(14.3)$ & $15(14.6)$ & .003 & A vs $C$ and $D$ \\
\hline Bilateral PEA & $285(82.1)$ & $66(79.5)$ & $53(75.7)$ & 80 (87.9) & $86(83.5)$ & .18 & - \\
\hline \multicolumn{8}{|l|}{ Operative } \\
\hline CPB time (min) & $308 \pm 96$ & $236 \pm 77$ & $310 \pm 103$ & $333 \pm 80$ & $344 \pm 87$ & $<.001$ & $\begin{array}{l}\mathrm{A} \text { vs } \mathrm{B}, \mathrm{C} \text {, and } \mathrm{D} \\
\mathrm{B} \text { vs } \mathrm{C} \text { and } \mathrm{D}\end{array}$ \\
\hline Hypothermia $\left({ }^{\circ} \mathrm{C}\right)$ & $21.0 \pm 3.4$ & $17.5 \pm 1.6$ & $17.9 \pm 2.1$ & $23.3 \pm 1.9$ & $24.0 \pm 0.8$ & $<.001$ & $\begin{array}{l}\text { A vs } C \text { and } D \\
B \text { vs } C \text { and } D\end{array}$ \\
\hline Number of HCA & $7.4 \pm 5.7$ & $2.0 \pm 0.7$ & $3.6 \pm 1.5$ & $8.9 \pm 3.9$ & $13.1 \pm 4.9$ & $<.001$ & $\begin{array}{l}\text { A vs } B, C \text { and } D \\
B \text { vs C and D } \\
\text { C vs D }\end{array}$ \\
\hline Total HCA time (min) & $64.2 \pm 39.7$ & $26.4 \pm 15.0$ & $50.6 \pm 28.9$ & $85.7 \pm 36.0$ & $84.9 \pm 36.0$ & $<.001$ & $\begin{array}{l}\mathrm{A} \text { vs } \mathrm{B}, \mathrm{C} \text { and } \mathrm{D} \\
\mathrm{B} \text { vs } \mathrm{C} \text { and } \mathrm{D}\end{array}$ \\
\hline
\end{tabular}

Data presented as mean \pm standard deviation, $\mathrm{n}(\%)$, or median and interquartile range. LOSD, Length of severe disease (World Health Organization class III or IV symptoms); $J 3$, Jamieson type $3 ; m P A P$, mean pulmonary arterial pressure; $P V R$, pulmonary vascular resistance; $P E A$, pulmonary endarterectomy; $C P B$, cardiopulmonary bypass; $H C A$, hypothermic circulatory arrest.

upper for LMV and total intensive care unit stay). Finally, we included a combined endpoint of none vs any of these endpoints. The absence of any outcome endpoint was considered uneventful outcome.

Stata, version 11 (StataCorp, College Station, Tex), was used for computation. All $P$ values were 2 -sided. Statistical significance was considered for $P<.05$. The Bonferroni correction was used for post hoc comparisons.

\section{RESULTS}

The patient characteristics were analyzed according to the 4 groups (Table 2). Over time, the patient age increased significantly (Figure 2). For the duration of severe disease, defined as the period from the onset of World Health Organization class III or IV symptoms, a great variability was observed. Even if the duration of severe disease assessment is determined by a patient's self-evaluation, it is evident that a late diagnosis remains an important issue. ${ }^{3}$ CTEPH was characterized intraoperatively according to Jamieson's classification. ${ }^{17} \mathrm{~J} 3$ lesions were considered the most challenging. A trend toward an increase in the number of $\mathrm{J} 3$ lesions, even if not statistically significant, reflects our learning curve. Hemodynamic impairment was more severe in patients in group A, early in our experience. Urgent PEA was defined as the need for surgery within 48 hours of the admission. Emergency PEA was defined as the need to bring the patient immediately to the operating room because of severe hemodynamic instability. A significantly greater proportion of PEAs were performed in urgent or emergency conditions in group A. This could explain the different presenting hemodynamic profile of the same group and highlights the delay in referring patients to surgery early in our experience. The need for oxygen therapy did not change over time, confirming that about $50 \%$ of patients with operable CTEPH experience chronic respiratory failure. ${ }^{3}$

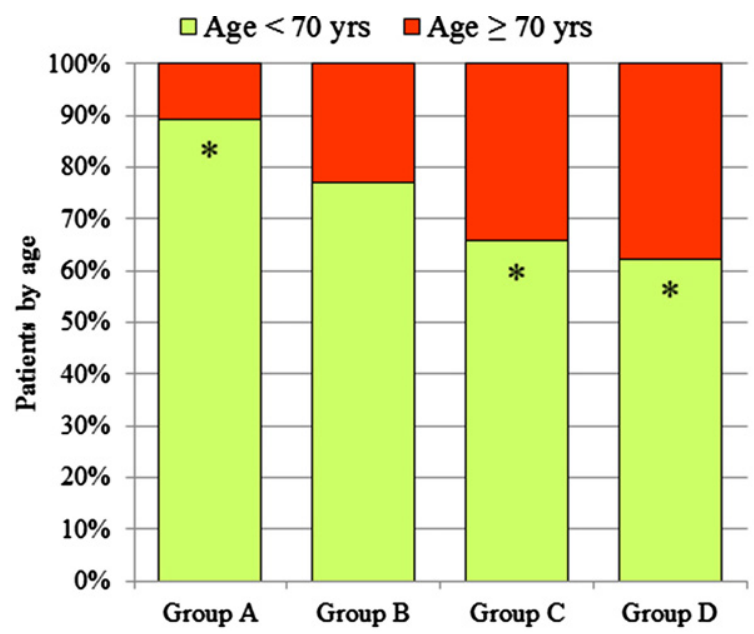

FIGURE 2. Population by group, dichotomized by age. Overall $P<.001$. *Significant after Bonferroni correction $(P<.008)$. 
TABLE 3. Early hemodynamic changes after pulmonary endarterectomy stratified by group

\begin{tabular}{|c|c|c|c|c|}
\hline Variable & Group A & Group B & Group C & Group D \\
\hline \multicolumn{5}{|l|}{ mPAP (mm Hg) } \\
\hline Preoperative & $50 \pm 13$ & $46 \pm 12$ & $41 \pm 12$ & $43 \pm 11$ \\
\hline Postoperative & $27 \pm 10$ & $21 \pm 8$ & $21 \pm 7$ & $23 \pm 8$ \\
\hline Change & $22 \pm 12(20-25)$ & $25 \pm 13(22-28)$ & $20 \pm 12(18-23)$ & $20 \pm 13(17-23)$ \\
\hline$P$ value & $<.001$ & $<.001$ & $<.001$ & $<.001$ \\
\hline \multicolumn{5}{|c|}{$\operatorname{PVR}\left(\right.$ dyne $\cdot \mathrm{s} \cdot \mathrm{cm}^{-5}$ ) } \\
\hline Preoperative & $1214 \pm 485$ & $1060 \pm 549$ & $877 \pm 421$ & $897 \pm 419$ \\
\hline Postoperative & $340 \pm 239$ & $270 \pm 181$ & $276 \pm 130$ & $319 \pm 202$ \\
\hline Change & $825 \pm 396(734-916)$ & $758 \pm 454(644-873)$ & $585 \pm 427(487-683)$ & $568 \pm 397(484-653)$ \\
\hline$P$ value & $<.001$ & $<.001$ & $<.001$ & $<.001$ \\
\hline
\end{tabular}

Data presented as mean \pm standard deviation ( $95 \%$ confidence interval). $m P A P$, Mean pulmonary arterial pressure; $P V R$, pulmonary vascular resistance.

An analysis of the operative parameters showed the changes made to our protocol (Table 2). Hypothermia changed from $18^{\circ} \mathrm{C}$ (groups A and B) to $24^{\circ} \mathrm{C}$ (groups C and $\mathrm{D}$ ). The number of HCA sessions increased significantly, as did the total HCA time and, consequently, the CPB time. Despite the greater number of HCA sessions in group D than in group C, the total HCA time did not differ, because each HCA session performed in group D was shorter.

The hemodynamics at discharge showed a remarkable improvement in each group (Table 3), with a mean pulmonary arterial pressure reduction of $20 \mathrm{~mm} \mathrm{Hg}$ or more and a PVR decrease of more than 560 dyne $\cdot \mathrm{s} \cdot \mathrm{cm}^{-5}$.

The univariate and multivariate outcome analysis results are summarized in Figure 3 and Table 4. Improved postoperative respiratory function was observed, expressed by a threefold increase in the likelihood of obtaining a $\mathrm{PaO}_{2} /$ $\mathrm{FiO}_{2} 6 \mathrm{~h}$ ratio greater than 217 for group $\mathrm{C}$ patients. The introduction of intermittent moderate HCA also resulted in a significant reduction in the LMV, with a threefold increase in the likelihood of extubation within postoperative day 4 for group D patients. Furthermore, patients in group D had a more than threefold decrease in the likelihood of presenting with any postoperative infection, perhaps because ventilator-associated pneumonia is still the most frequent infective complication after PEA., ${ }^{3,6,18,19}$ Finally, for patients in groups $\mathrm{C}$ and $\mathrm{D}$, a remarkable increase in the likelihood of having an uneventful outcome was observed, three- to fourfold, respectively. In concert with the development of our alternative CPB strategy, a trend toward a reduction in the incidence of transient neurologic events was observed. The overall incidence of permanent neurologic events (6/347) was too low for any comparison. In our experience, the different CPB strategies influenced neither operative nor hospital mortality, which were all in line with previously reported data. ${ }^{3,6,11,17-20}$

\section{DISCUSSION}

A detailed preoperative patient evaluation, appropriate selection, surgical technique and experience, and meticulous postoperative management are essential prerequisites for success after PEA. The selection of patients for surgery depends on the extent and location of the organized thrombi in relation to the degree of pulmonary hypertension. Proximal organized lesions represent the ideal situation, although the surgical indication for more distal obstructions can be quite different, according to the surgeon's expertise. The lung ventilation/perfusion scan is still the most sensitive test for the diagnosis of CTEPH, showing even small ventilation/perfusion mismatches. For operability assessment, the most powerful diagnostic tool is high-resolution, 64-row angiographic computed tomography, which provides many useful details about the pulmonary arterial wall and lumen, heart morphology, lung parenchyma, and mediastinum. ${ }^{25}$ The degree of pulmonary hypertension must be assessed by right heart catheterization, both at rest and during exercise, to diagnose those patients with normal hemodynamics at rest but exertional pulmonary hypertension.

We believe the most relevant challenge in CTEPH remains the diagnosis. Misdiagnosis and late diagnosis lead to low patient referral and, subsequently, a slow learning curve. At the beginning of our experience with PEA, our referral was limited to severely compromised patients, and about $30 \%$ of PEAs were performed as urgent or emergency operations. The referring physicians were reticent to suggest a surgical evaluation of their elderly patients, because PEA was still considered an extremely high-risk operation. After adopting the intermittent HCA technique as the procedure of choice, we could safely perform PEA even in the elderly. As our surgical results were not affected by including patients older than 70 years, the referral of the elderly to our center increased, reflecting an increased awareness of the disease and its effective surgical treatment among the referring physicians. Also, our alternative technique allowed us to take more time to explore thoroughly even the segmental branches found to be patent on the angiographic computed tomography scan. Some unexpected lesions such as recanalized thrombi and webs, which can 

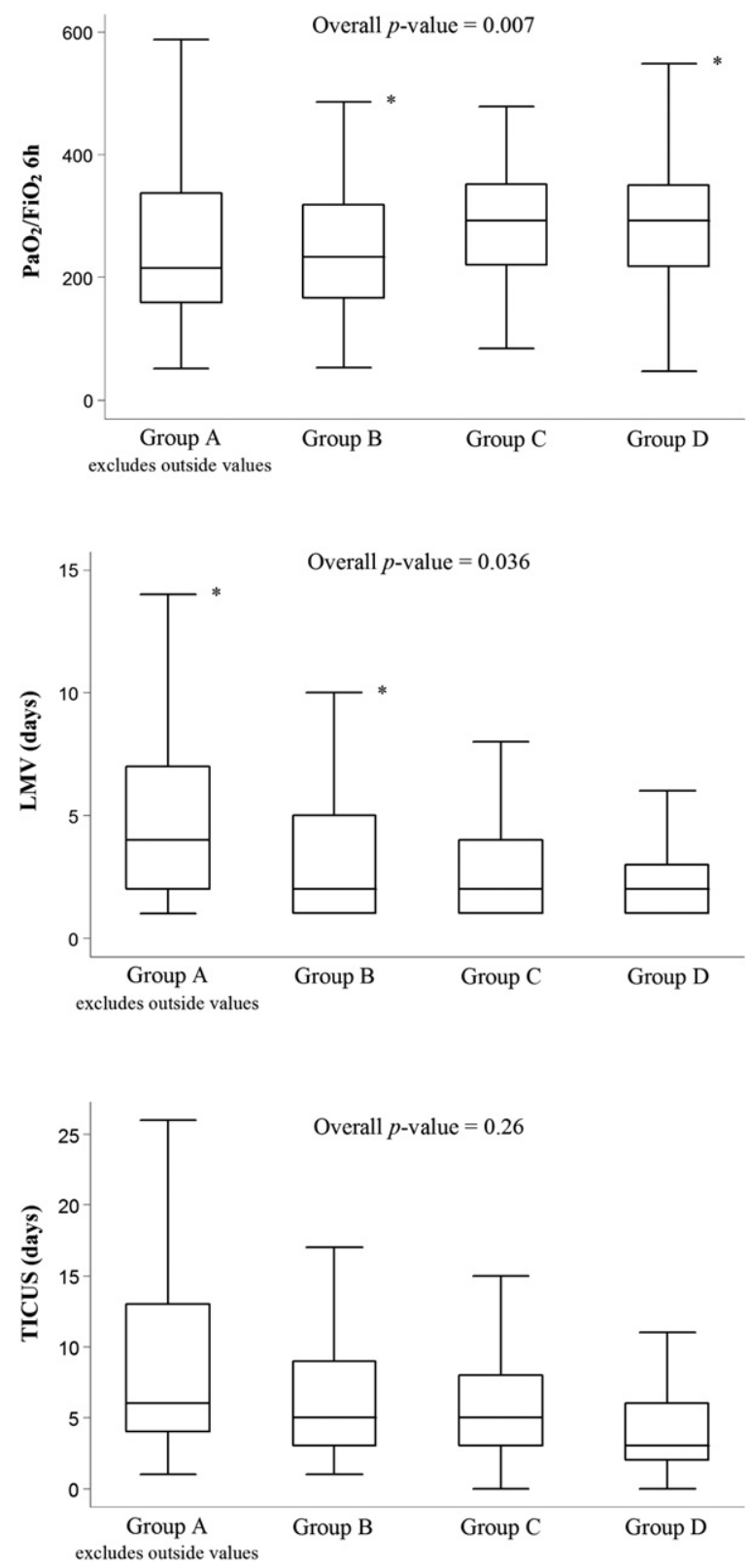

FIGURE 3. Continuous univariate outcome analysis summarized in box plot diagrams. $\mathrm{PaO}_{2} / \mathrm{FiO}_{2} 6 \mathrm{~h}$, Ratio between partial pressure of oxygen in arterial blood and fraction of inspired oxygen measured 6 hours after admission to intensive care unit; $L M V$, length of mechanical ventilation; TICUS, total intensive care unit stay. *Significant after Bonferroni correction $(P<.008)$.

be regularly perfused by the contrast agent, were intraoperatively detected and successfully removed. This improved our collaboration with our radiologist, who created an archive of the most distal PEA procedures, updated periodically. During the past 3 years, we have witnessed an increase in the number of patients referred with CTEPH, and we have gained experience to extend the surgical indication to more distal disease. Hemodynamic improvement after PEA was remarkable for each group, despite a trend toward an increase in the number of $\mathrm{J} 3$ lesions.

Although we extended the surgical indication to more challenging CTEPH,${ }^{18}$ and although the patients undergoing PEA were becoming older over time, the outcomes improved. Our multivariate analysis showed that the introduction of intermittent moderate HCA with periods of 7 minutes at most resulted in improved postoperative respiratory function, a reduced LMV, a reduction in the incidence of infection, and a remarkable improvement in the likelihood of uneventful outcomes.

Retrospective studies are particularly useful in investigating diseases of low incidence, but any retrospective study is subject to selection bias. There is no reason to believe that the epidemiology of CTEPH has changed over time. The increase in the number of patients referred with CTEPH was rather owing to a recent change in the referring physicians' attitudes. The intervention, baseline state, and outcomes were obtained from existing recorded information. However, our study included all consecutive incident patients. Our database was not modified over time, and all variables were collected prospectively. Our case groups were identified from the same population of operated CTEPH patients. The groups were noncontemporaneous owing to the study design. Thus, our learning curve, as well as the changes made in the patients' nonoperative management over time, could not be separated from the sequential changes in CPB management. However, although some outcome variables are clearly dependent on the surgeon's learning curve, such as the postoperative hemodynamics, other variables depend most on hypothermia and circulatory arrest, as neurologic events. In our experience, no differences in the PVR decrease after PEA were observed among the 4 groups, with a postoperative PVR always lower than $350 \mathrm{dyn} \cdot \mathrm{s} \cdot$ $\mathrm{cm}^{-5}$, showing that the surgeon's learning curve resulted mainly in the ability to perform PEA for more distal disease. That this was possible was definitely because of our new CPB strategy, which resulted in us being able to significantly and safely prolong the total HCA time. What changed in the patients' outcome after adopting our alternative technique was the absence of any transient neurologic event, such as choreic movements, psychomotor agitation, late awakening, or late extubation due to asthenia. The consequent fast track recovery with early extubation, sometimes even less than 24 hours, resulted in better postoperative respiratory function, a lower infections rate, and a remarkable increase in the likelihood of a completely uneventful outcome. Despite the longer total HCA and CPB times, operative and hospital mortality were not affected. We therefore believe that the improvement in patients' outcomes mainly resulted from the sequential changes in the CPB management and cerebral protection strategy, suggesting a protective role for our alternative strategy on the central nervous system during surgery. 
TABLE 4. Categorical univariate and multivariate outcome analysis

\begin{tabular}{|c|c|c|c|c|c|c|c|}
\hline \multirow[b]{2}{*}{ Group } & \multirow[b]{2}{*}{ Patients (n) } & \multicolumn{3}{|c|}{ Univariate } & \multicolumn{3}{|c|}{ Multivariate* } \\
\hline & & OR $(95 \%$ CI $)$ & $P$ value & Other comparisons & OR $(95 \%$ CI $)$ & $P$ value & Other comparisons \\
\hline $\mathrm{PaO}_{2} / \mathrm{FiO}_{2} 6 \mathrm{~h} \leq 217$ & & & $<.001$ & & & .01 & \\
\hline A & $38(50.7)$ & 1 & & & 1 & & \\
\hline B & $29(43.3)$ & $0.74(0.38-1.44)$ & .38 & $\mathrm{~B}$ vs $\mathrm{C}, P=.004$ & $0.8(0.39-1.65)$ & .55 & $\mathrm{~B}$ vs $\mathrm{C}, P=.02$ \\
\hline $\mathrm{C}$ & $19(21.6)$ & $0.27(0.14-0.53)$ & $<.001$ & $\mathrm{~B}$ vs $\mathrm{D}, P=.01$ & $0.33(0.15-0.71)$ & .005 & $\mathrm{~B}$ vs $\mathrm{D}, P=.06$ \\
\hline $\mathrm{D}$ & $25(24.8)$ & $0.32(0.17-0.61)$ & $<.001$ & $\mathrm{C}$ vs $\mathrm{D}, P=.61$ & $0.40(0.19-0.85)$ & .02 & $\mathrm{C}$ vs $\mathrm{D}, P=.61$ \\
\hline $\mathrm{LMV} \geq 4 \mathrm{~d}$ & & & $<.001$ & & & .03 & \\
\hline A & $42(50.6)$ & 1 & & & 1 & & \\
\hline $\mathrm{B}$ & $21(30)$ & $0.42(0.21-0.82)$ & .01 & $\mathrm{~B}$ vs $\mathrm{C}, P=.88$ & $0.44(0.21-0.92)$ & .03 & $\mathrm{~B}$ vs $\mathrm{C}, P=.37$ \\
\hline $\mathrm{C}$ & $28(31.1)$ & $0.44(0.24-0.82)$ & .01 & $\mathrm{~B}$ vs $\mathrm{D}, P=.21$ & $0.62(0.3-1.27)$ & .19 & $\mathrm{~B}$ vs $\mathrm{D}, P=.59$ \\
\hline $\mathrm{D}$ & $22(21.6)$ & $0.27(0.14-0.51)$ & $<.001$ & $\mathrm{C}$ vs $\mathrm{D}, P=.14$ & $0.35(0.17-0.73)$ & .005 & $\mathrm{C}$ vs $\mathrm{D}, P=.14$ \\
\hline TICUS $\geq 7 \mathrm{~d}$ & & & .18 & & & .49 & \\
\hline A & $32(38.6)$ & 1 & & & 1 & & \\
\hline B & $25(35.7)$ & $0.89(0.46-1.71)$ & .72 & - & $0.85(0.41-1.75)$ & .66 & - \\
\hline $\mathrm{C}$ & $30(33)$ & $0.78(0.42-1.46)$ & .44 & - & $1.06(0.52-2.17)$ & .87 & - \\
\hline $\mathrm{D}$ & $25(24.3)$ & $0.51(0.27-0.96)$ & .04 & - & $0.65(0.32-1.33)$ & .24 & - \\
\hline Reperfusion edema & & & $<.001$ & & & .001 & \\
\hline A & $17(23)$ & 1 & & & 1 & & \\
\hline B & $4(5.7)$ & $0.21(0.07-0.65)$ & .007 & $\mathrm{~B}$ vs $\mathrm{C}, P=.46$ & $0.08(0.02-0.32)$ & $<.001$ & B vs $\mathrm{C}, P=.61$ \\
\hline $\mathrm{C}$ & $3(3.3)$ & $0.12(0.03-0.41)$ & .001 & B vs $\mathrm{D}, P=.78$ & $0.04(0.003-0.53)$ & .02 & B vs $\mathrm{D}, P=.15$ \\
\hline $\mathrm{D}$ & $7(6.9)$ & $0.25(0.1-0.63)$ & .004 & $\mathrm{C}$ vs $\mathrm{D}, P=.28$ & $0.25(0.07-0.92)$ & .04 & $\mathrm{C}$ vs $\mathrm{D}, P=.11$ \\
\hline Tracheostomy & & & .84 & & & .88 & \\
\hline A & $7(8.4)$ & 1 & & & 1 & & \\
\hline B & $4(5.7)$ & $0.66(0.18-2.35)$ & .52 & - & $0.73(0.18-3.04)$ & .67 & - \\
\hline $\mathrm{C}$ & $5(5.5)$ & $0.63(0.19-2.08)$ & .45 & - & $0.84(0.18-3.92)$ & .83 & - \\
\hline $\mathrm{D}$ & $8(7.8)$ & $0.91(0.32-2.64)$ & .87 & - & $1.22(0.35-4.23)$ & .75 & - \\
\hline Infections & & & .006 & & & .02 & \\
\hline A & $18(21.7)$ & 1 & & & 1 & & \\
\hline B & $25(35.7)$ & $2(0.98-4.11)$ & .06 & $\mathrm{~B}$ vs $\mathrm{C}, P=.15$ & $1.9(0.86-4.17)$ & .11 & $\mathrm{~B}$ vs $\mathrm{C}, P=.52$ \\
\hline $\mathrm{C}$ & $23(25.3)$ & $1.22(0.6-2.47)$ & .58 & $\mathrm{~B}$ vs $\mathrm{D}, P<.001$ & $1.48(0.65-3.37)$ & .35 & B vs $\mathrm{D}, P=.004$ \\
\hline $\mathrm{D}$ & $13(12.5)$ & $0.52(0.24-1.13)$ & .10 & $\mathrm{C}$ vs $\mathrm{D}, P=.02$ & $0.57(0.24-1.36)$ & .20 & $\mathrm{C}$ vs $\mathrm{D}, P=.02$ \\
\hline TNE & & & .19 & & & .32 & \\
\hline A & $13(17.6)$ & 1 & & & 1 & & \\
\hline B & $10(14.9)$ & $0.82(0.33-2.03)$ & .67 & - & $0.81(0.31-2.13)$ & .67 & - \\
\hline $\mathrm{C}$ & $9(10.7)$ & $0.56(0.23-1.41)$ & .22 & - & $0.63(0.24-1.67)$ & .35 & - \\
\hline $\mathrm{D}$ & $7(7.1)$ & $0.36(0.14-0.96)$ & .04 & - & $0.34(0.1-1.08)$ & .07 & - \\
\hline Operative mortality & & & .77 & & & .53 & \\
\hline A & $9(10.8)$ & 1 & & & 1 & & \\
\hline B & $5(7.1)$ & $0.63(0.2-1.99)$ & .43 & - & $0.36(0.07-1.91)$ & .23 & - \\
\hline $\mathrm{C}$ & $8(8.8)$ & $0.79(0.29-2.16)$ & .65 & - & $1.26(0.38-4.1)$ & .71 & - \\
\hline $\mathrm{D}$ & $7(6.8)$ & $0.6(0.21-1.69)$ & .33 & - & $0.9(0.29-2.79)$ & .85 & - \\
\hline Hospital mortality & & & .91 & & & .49 & \\
\hline A & $10(12.1)$ & 1 & & & 1 & & \\
\hline B & $6(8.6)$ & $0.68(0.24-1.99)$ & .49 & - & $0.43(0.11-1.63)$ & .21 & - \\
\hline $\mathrm{C}$ & $9(9.9)$ & $0.80(0.31-2.08)$ & .65 & - & $1.17(0.39-3.51)$ & .78 & - \\
\hline $\mathrm{D}$ & $10(9.7)$ & $0.78(0.31-1.99)$ & .61 & - & $1.05(0.38-2.89)$ & .93 & - \\
\hline Uneventful outcome & & & $<.001$ & & & .001 & \\
\hline A & $20(24.1)$ & 1 & & & 1 & & \\
\hline B & $22(31.4)$ & $1.44(0.71-2.95)$ & .31 & B vs $\mathrm{C}, P=.04$ & $1.39(0.64-3.02)$ & .41 & $\mathrm{~B}$ vs $\mathrm{C}, P=.21$ \\
\hline $\mathrm{C}$ & $43(47.3)$ & $2.82(1.47-5.41)$ & .002 & $\mathrm{~B}$ vs $\mathrm{D}, P<.001$ & $2.21(1.06-4.64)$ & .04 & $\mathrm{~B}$ vs $\mathrm{D}, P=.003$ \\
\hline $\mathrm{D}$ & $64(60.4)$ & $4.8(2.54-9.08)$ & $<.001$ & $\mathrm{C}$ vs $\mathrm{D}, P=.07$ & $4.05(1.95-8.39)$ & $<.001$ & $\mathrm{C}$ vs $\mathrm{D}, P=.07$ \\
\hline
\end{tabular}

$\mathrm{OR}$, Odds ratio; $\mathrm{CI}$, confidence interval; $\mathrm{PaO}_{2} / \mathrm{FiO}_{2} 6 \mathrm{~h}$, ratio between partial pressure of oxygen in arterial blood and fraction of inspired oxygen measured 6 hours after admission to intensive care unit; $L M V$, length of mechanical ventilation; TICUS, total intensive care unit stay; TNE, transient neurologic event. *Adjusted for age, duration of severe disease (LOSD), Jamieson type 3 chronic thromboembolic pulmonary hypertension lesions, preoperative mean pulmonary arterial pressure and pulmonary vascular resistance, and pulmonary endarterectomy surgical timing. 


\section{CONCLUSIONS}

To achieve a bloodless surgical field to perform a complete PEA, HCA is still considered mandatory. The purpose of our study was to show the effectiveness of an alternative CPB management for PEA developed at our center during a 17-year experience. Our research has shown how our new technique cannot only reduce the invasiveness of such demanding surgery, especially for the elderly, but also extend the surgical indication to patients with exclusively $\mathrm{J} 3$ lesions.

In our experience, this new protocol represents an effective alternative to the original technique, allowing for a better combination of surgical accuracy and patient outcomes. Adopting this strategy, PEA can be performed safely despite a longer total HCA time, thus giving more time for the accurate dissection of the distal branches. With the combination of moderate hypothermia with intermittent systemic reperfusion, the patient outcomes were improved, despite treating older patients and more distal disease.

We believe that our described CPB management could be useful, especially for those centers planning to start a PEA program, providing the possibility of safely performing surgery with a longer HCA time. This could be particularly helpful for lung transplant centers, where a PEA program could maximize the use of scarce organ donors by offering, when feasible, a nontransplant option to patients with CTEPH. As an ethical consideration, however, it remains mandatory to fulfill all the criteria for the definition of an expert center, as reported in the European guidelines, ${ }^{16}$ to offer patients the best treatment and to achieve the best results.

We would like to express our gratitude to Professor Thomas M. Egan, MD, MSc, from the University of North Carolina at Chapel Hill (Chapel Hill, NC) for editorial revision of our report.

\section{References}

1. Condliffe R, Kiely DG, Gibbs JS, Corris PA, Peacock AJ, Jenkins DP, et al. Improved outcomes in medically and surgically treated chronic thromboembolic pulmonary hypertension. Am J Respir Crit Care Med. 2008;177:1122-7.

2. Piazza G, Goldhaber SZ. Chronic thromboembolic pulmonary hypertension. $N$ Engl J Med. 2011;364:351-60.

3. Mayer E, Jenkins D, Lindner J, D'Armini A, Kloek J, Meyns B, et al. Surgical management and outcome of patients with chronic thromboembolic pulmonary hypertension: results from an international prospective registry. J Thorac Cardiovasc Surg. 2011;141:702-10.

4. Archibald CJ, Auger WR, Fedullo PF, Channick RN, Kerr KM, Jamieson SW, et al. Long-term outcome after pulmonary thromboendarterectomy. Am J Respir Crit Care Med. 1999;160:523-8.

5. Reesink HJ, van der Plas MN, Verhey NE, van Steenwijk RP, Kloek JJ, Bresser P. Six-minute walk distance as parameter of functional outcome after pulmonary endarterectomy for chronic thromboembolic pulmonary hypertension. J Thorac Cardiovasc Surg. 2007;133:510-6.
6. Bonderman D, Skoro-Sajer N, Jakowitsch J, Adlbrecht C, Dunkler D, Taghavi S, et al. Predictors of outcome in chronic thromboembolic pulmonary hypertension. Circulation. 2007; 115:2153-8.

7. Jamieson SW, Auger WR, Fedullo PF, Channick RN, Kriett JM, Tarazi RY, et al. Experience and results with 150 pulmonary thromboendarterectomy operations over a 29-month period. J Thorac Cardiovasc Surg. 1993;106:116-26.

8. D’Armini AM, Zanotti G, Ghio S, Magrini G, Pozzi M, Scelsi L, et al. Reverse right ventricular remodeling after pulmonary endarterectomy. J Thorac Cardiovasc Surg. 2007; 133:162-8

9. Corsico AG, D’Armini AM, Cerveri I, Klersy C, Ansaldo E, Niniano R, et al. Long-term outcome after pulmonary endarterectomy. Am J Respir Crit Care Med. 2008;178:419-24.

10. Riedel M, Stanek V, Widimsky J, Prerovsky I. Longterm follow-up of patient with pulmonary thromboembolism: late prognosis and evolution of hemodynamic and respiratory data. Chest. 1982;81:151-8.

11. Fedullo PF, Auger WR, Kerr KM, Rubin LJ. Chronic thromboembolic pulmonary hypertension. $N$ Engl J Med. 2001;345:1465-72.

12. Pengo V, Lensing AW, Prins MH, Marchiori A, Davidson BL, Tiozzo F, et al. Incidence of chronic thromboembolic pulmonary hypertension after pulmonary embolism. N Engl J Med. 2004;350:2257-64.

13. Dentali F, Donadini M, Gianni M, Bertolini A, Squizzato A, Venco A, et al. Incidence of chronic pulmonary hypertension in patients with previous pulmonary embolism. Thromb Res. 2009;124:256-8.

14. Galiè N, Kim NH. Pulmonary microvascular disease in chronic thromboembolic pulmonary hypertension. Proc Am Thorac Soc. 2006;3:571-6.

15. Arbustini E, Morbini P, D'Armini AM, Repetto A, Minzioni G, Piovella F, et al. Plaque composition in plexogenic and thromboembolic pulmonary hypertension: the critical role of thrombotic material in pultaceous core formation. Heart. 2002; 88:177-82.

16. Galiè N, Hoeper MM, Humbert M, Torbicki A, Vachiery JL, Barbera JA, et al Guidelines for the diagnosis and treatment of pulmonary hypertension: the Task Force for the Diagnosis and Treatment of Pulmonary Hypertension of the European Society of Cardiology (ESC) and the European Respiratory Society (ERS), endorsed by the International Society of Heart and Lung Transplantation (ISHLT). Eur Heart J. 2009;30:2493-537.

17. Thistlethwaite PA, Mo M, Madani MM, Deutsch R, Blanchard D, Kapelanski DP, et al. Operative classification of thromboembolic disease determines outcome after pulmonary endarterectomy. J Thorac Cardiovasc Surg. 2002; $124: 1203-11$

18. Thistlethwaite PA, Kemp A, Du L, Madani MM, Jamieson SW. Outcomes of pulmonary endarterectomy for treatment of extreme thromboembolic pulmonary hypertension. J Thorac Cardiovasc Surg. 2006;131:307-13.

19. Jamieson SW, Kapelanski DP, Sakakibara N, Manecke GR, Thistlethwaite PA Kerr KM, et al. Pulmonary endarterectomy: experience and lessons learned in 1,500 cases. Ann Thorac Surg. 2003;76:1457-64

20. Freed DH, Thomson BM, Berman M, Tsui SS, Dunning J, Sheares KK, et al. Survival after pulmonary thromboendarterectomy: effect of residual pulmonary hypertension. J Thorac Cardiovasc Surg. 2011;141:383-7.

21. Macchiarini P, Kamiya H, Hagl C, Winterhalter M, Barbera J, Karck M, et al. Pulmonary endarterectomy for chronic thromboembolic pulmonary hypertension: is deep hypothermia required? Eur J Cardiothorac Surg. 2006;30:237-43.

22. Thomson B, Tsui SS, Dunning J, Goodwin A, Vuylsteke A, Latimer R, et al. Pulmonary endarterectomy is possible and effective without the use of complete circulatory arrest - the UK experience in over 150 patients. Eur J Cardiothorac Surg. 2008;33:157-63.

23. D'Armini AM, Zanotti G, Viganò M. Pulmonary endarterectomy: the treatmen of choice for chronic thromboembolic pulmonary hypertension. Ital Heart J. 2005;6:861-8.

24. D'Armini AM, Cattadori B, Monterosso C, Klersy C, Emmi V, Piovella F, et al. Pulmonary thromboendarterectomy in patients with chronic thromboembolic pulmonary hypertension: hemodynamic characteristics and changes. Eur J Cardiothorac Surg. 2000;18:696-702.

25. Sundt TM, Williamson EE. High-resolution computed tomography with threedimensional reconstruction for assessment of chronic pulmonary thromboembolic disease. J Thorac Cardiovasc Surg. 2011;141:1539-40. 